Cahiers de recherches médiévales

\title{
Le statut philosophique des communes médiévales chez Saint-Simon, A. Thierry et A. Comte
}

Laurent Clauzade

\section{(2) OpenEdition}

Édition électronique

URL : https://journals.openedition.org/crm/2488

DOI : $10.4000 / \mathrm{crm} .2488$

ISSN : 1955-2424

Éditeur

Honoré Champion

\section{Édition imprimée}

Date de publication : 12 décembre 1996

Pagination : 97-107

ISSN : $1272-9752$

\section{Référence électronique}

Laurent Clauzade, «Le statut philosophique des communes médiévales chez Saint-Simon, A. Thierry et A. Comte », Cahiers de recherches médiévales [En ligne], 2 | 1996, mis en ligne le 04 février 2008, consulté le 15 décembre 2022. URL : http://journals.openedition.org/crm/2488 ; DOI : https://doi.org/ $10.4000 / \mathrm{crm} .2488$ 


\section{RM}

\section{Le statut philosophique des communes médiévales chez Saint- Simon, A. Thierry et A. Comte.}

A la question «comment terminer la Révolution?" qui obsède la réflexion politique sous la Restauration, répond en écho une autre question, plus historique peut-être, mais tout aussi idéologique: quand la Révolution a-t-elle véritablement commencée? Les événements de 1789 sont alors compris comme le point d'aboutissement, ou comme une étape particulièrement importante, d'un mouvement plus ancien, destiné à abattre l'ordre politique mis en place par le Moyen Age. Dans cette perspective, l'affranchissement des communes serait la première manifestation de cette série destinée à former une ère politique nouvelle.

Nous nous proposons d'étudier cette thèse chez trois penseurs dont la réflexion sur l'histoire est un lieu privilégié pour la construction de leur système: Saint-Simon, Augustin Thierry et Auguste Comte. Les deux demiers auteurs ont pour point commun d'avoir été successivement secrétaires de Saint-Simon à une période, dite " industrielle ", où ce dernier élabore le thème de l'affranchissement des communes. Si les analyses des anciens élèves vont alors apporter de notables variations à la thèse saint-simonienne, toutes trois tentent, en définitive, de saisir quel est le véritable lien entre le Moyen Age et l'époque moderne.

\section{Saint-Simon.}

Le thème de l'affranchissement des communes apparaît dans l'œuvre de Saint-Simon autour de 1817. A cette époque le publiciste a abandonné ses spéculations sur les sciences, pour se consacrer à la promotion du pouvoir industriel. Cette période de sa vie est aussi celle de sa collaboration avec Augustin Thierry, laquelle, commencée vers 1814, s'achèvera avec la publication de l'Industrie.

C'est justement dans la partie de cet ouvrage consacrée à la politique et rédigée par A. Thierry, que l'affranchissement des communes est présenté comme une date charnière de l'histoire de l'humanité:

Le caractère des peuples de l'antiquité était essentiellement militaire. Ce qu'il y avait de travail paisible était rejeté hors de la nation et abandonné aux esclaves. La grande industrie, c'était la guerre; avec l'épée on produisait, par l'épée se grossissaient les richesses de l'Etat et des particuliers.

Cet ordre de choses devait finir avec l'état moral qui l'avait produit et qui le maintenait; un autre devait lui succóder, conforme à cette direction intérieure, qui remplaçait dans l'homme son ancienne tendance à se jeter hors de lui. La révolution se fit dans le XII siècle.

Par l'affranchissement des communes dans toute l'Europe, l'industrie paisible, qui, chez les anciens, était hors de l'Etat, entra dans l'Etat, et en devint une partie active, de passive qu'elle était d'abord. 


\section{Laurent CLAUZADE}

A cet événement se rattachent à la fois l'époque de la liberté des services et l'époque de la paix, devenues un objet de la politique; deux changements, d'où sont venues en Europe toutes les révolutions passées, et d'où sortimont toutes celles qui sont encore à naitre.'

Les lignes de force de l'analyse du pouvoir temporel, chez Saint-Simon comme chez Auguste Comte, sont dejà présentes dans cette première évocation. Ce qui se joue au XII $\mathrm{s}$. et à la fin du Moyen Age, c'est la radicale transformation du pouvoir temporel : de militaire et guerrier, il devient industriel et pacifique. L'affranchissement des communes marque donc le passage d'un pouvoir à l'autre. En d'autres termes, alors que le système féodal est la dernière manifestation du pouvoir militaire, l'industrie commence véritablement à se constituer comme force politique à partir du $\mathrm{Xn}^{\mathrm{e}} \mathrm{s}$. : elle n'est plus alors " hors de l'Etat ", mais « dans l'Etat».

Cela ne signifie pas que la classe industrielle n'existait pas auparavant : elle n'avait seulement aucun rôle politique, son statut étant par définition la négation même de toute puissance temporelle. C'est ce que précise le quatrième tome de l'Industrie signé seulement par Saint-Simon :

Si l'on remonte dans l'histoire de l'industrie jusqu'à l'époque des Grecs et des Romains, on trouve que chez ces peuples, la classe industrielle était complètement esclave de la classe militaire.

L'esclavage de l'industrie continua sous les guerriers du Nord qui détruisirent l'empire romain, et qui s'établirent dans l'occident de l'Europe, à la place des anciens maitres ou vainqueurs. ${ }^{2}$

Le terme d'affranchissement correspond donc à une opération bien précise : il s'agit pour l'industrie, en rachetant sa liberté, de passer de l'esclavage à l'autonomie politique, quel qu'en soit le degré initial :

«Les avantages que la destruction de l'empire romain procura (...) à l'industrie, lui ayant permis de prendre un certain développement, elle parvint graduellement au point de pouvoir racheter sa liberté. Ce rachat est le plus important de tous les pas que l'industrie ait faits et de tous ceux qu'elle fera par la suite. C'est le point le plus capital pour elle; c'est le commencement de son existence politique (...). . $)^{3}$

Cette analyse de la situation initiale montre qu'il ne faut pas restreindre le terme de " commune " à sa seule signification locale et administrative : ce qui est en jeu c'est fondamentalement le statut d'une classe sociale. C'est ce qu'indique d'ailleurs l'analyse du terme de «commune». A proprement parler, les communes et l'industrie sont une seule et même chose :

Ce pas est désigné ordinairement sous le nom d'affranchissement des communes; et c'est à bon droit qu'on se sert de cette expression, car les communes et l'industrie sont une seule et même chose: les communes, à leur origine, étant composées, en totalité, d'artisans et de négo-

\footnotetext{
${ }^{1} L$ 'Industrie (tome I, seconde partie, 1817), (Euvres de Claude-Henri de Saint-Simon, Genève, Slatkine reprints, 1977, t.I, p. 38-40.

${ }^{2} L$ 'Industrie (tome IV, seconde partie, 1818), op. cit. tII, p. 142.

${ }^{3}$ Ibid. p. 143.
} 
ciants qui s'étaient établis dans les villes. C'est là un fait très-essentiel à remarquer et qu'il ne faut jamais perdre de vue, pour se faire une idée juste de ce que nous devons entendre alljourd'hui par les communes. ${ }^{4}$

Même si le tome de l'Industrie d'où est tiré ce passage n'est pas signé par A. Thierry, on peut reconnaître dans cet essai de définition le souci constant qu'aura l'historien de rendre aux termes anciens leur véritable signification. Toujours est-il, cependant, que cette définition est plus sociologique, pour employer un mot qui n'existe pas encore, que proprement historique : les événements ne sont plus seulement le lieu d'inscription des actions des individus, ou des masses mues par des individus; l'histoire est aussi et surtout le terrain où s'affrontent des classes qui agissent en tant que telles, selon une logique propre que le sociologue, ou l'homme politique se doit de comprendre pour acquérir la "connaissance exacte des vrais éléments du présent " ${ }^{5}$. C'est pourquoi la stricte équivalences des notions d' « industrie " et de "commune", est un «fait». Cette analyse de vocabulaire montre bien que cette sociologie dont Saint-Simon fut l'inspirateur et que la philosophie positive d'Auguste Comte portera à son plein achèvement est fondamentalement une philosophie de l'histoire.

L'affranchissement des communes - ou encore le rachat par l'industrie de sa liberté - est donc le moment où la classe industrielle émerge comme force politique. Les premières élaborations que l'on trouve chez Saint-Simon restent très floues sur la chronologie, ou sur la correspondance entre les transformations sociales et les périodes historiques. Cette chronologie se précisera plus tard dans les œuvres d'A. Thierry et de Comte. Mais, dans l'Industrie, il n'est pas fait explicitement référence au Moyen Age. Tout au plus est-il question de la classe militaire ou féodale, des prêtres et des nobles. Le paradoxe qui consiste à faire émerger une force temporelle nouvelle au moment même où le système temporel et spirituel du Moyen Age atteint son développement intégral, n'apparaît donc pas dans les développements de Saint-Simon.

Néanmoins on trouve dans l'Industrie une première explication du rôle du Moyen Age dans l'affranchissement des communes. Mais cette réponse reste imparfaite pour deux raisons essentielles. Elle n'envisage tout d'abord que l'aspect temporel de la question, à savoir la puissance militaire ou industrielle. Ensuite cette tentative d'explication montre que l'affranchissement s'est fait plutôt malgré le système du Moyen Age, que grâce à lui :

Cette révolution [celle qui fait passer la classe industrielle de la domination romaine à celle des guerriers du nord], qui parait, au premier abord, n'avoir consisté pour l'industrie que dans un simple changement de maitre, fut cependant d'une grande importance, à raison des suites heureuses de ce changement de domination.

L'esclavage de la classe industrielle changea de nature et devint esclavage de la glèbe, ce qui était une grande amélioration. En outre, les vainqueurs s'étant répandus dans la campagne, les industriels, qui étaient établis dans les villes, ne furent plus soumis à l'inspection immédiate et continue des maitres, ce qui leur fut encore très-favorable.

${ }^{4}$ Ibid, 140. 
Ainsi, pour ces deux motifs, la conquête de l'empire romain par les peuples du nord de l'Eumpe produisit le premier perfectionnement qui ait eu lieu dans le sort de l'industrie. ${ }^{6}$

Ce perfectionnement entraîna un certain développement qui permit aux communes de racheter leur liberté. Dans ce scénario, le système féodal ne joue donc aucun rôle : c'est plutôt par négligence, par l'abandon des villes par les vainqueurs, que le phénomène des communes a pu se produire. Cette analyse appelle plusieurs remarques.

- Elle plaide tout d'abord pour la spontanéité du mouvement d'affranchissement: il n'est en aucun cas positivement tributaire du système temporel dans lequel il se produit.

- En second lieu, ce qu'implique cette spontanéité, c'est une radicale indépendance des deux systèmes, qui se côtoient sans s'influencer. Saint-Simon pense donc le Moyen Age, et en particulier la féodalité, comme radicalement hostile à l'industrie, au nom du principe militaire qui est censé lui donner son organisation.

- Enfin, la spontanéité et l'indépendance du mouvement des communes vis-à-vis du Moyen Age, conduit à penser l'industrie à partir de sa situation antérieure, à savoir l'époque des Grecs et des Romains. Vierge de toute influence médiévale, la classe industrielle apparaît telle qu'elle était dans l'antiquité. L'affranchissement est d'ailleurs présenté comme le passage de l'esclavage à l'existence politique. Un des enjeux des analyses qui suivront sera justement de déterminer, quelle que soit la signification qu'on lui donne, l'importance et le caractère de l'influence du modèle romain sur ce mouvement d'affranchissement.

\section{A. Thierry.}

Les Lettres Sth l'histoire de France, d'A. Thierry, paraissent en 1827, dix ans après sa collaboration avec Saint-Simon. Environ la moitie de cet ouvrage - onze lettres sur vingtcinq - est consacrée à l'affranchissement des communes. Dans ses grandes lignes son analyse reste fidèle à celle de l'Industrie; pourtant ni les enjeux ni la méthode ne sont les mêmes.

A. Thierry, en effet, ne se veut plus publiciste, ou essayiste politique, mais historien. Aussi ses lettres n'auront-elles plus la forme schématique des analyses précédentes:

Il me reste à parler de la méthode que j'ai suivie dans la composition de ces Lettres. La plupart sont des dissertations entremêlées de récits et de fragments des historiens originaux (...). Dans les matières historiques la méthode d'exposition est toujours la plus sûre, et ce n'est pas sans danger pour la vérité qu'on y introduit les subtilités de l'argumentation logique. C'est pour me conformer à ce principe que j'ai insisté avec tant de détails sur l'histoire politique de quelques villes politiques de France. Je voulais mettre en évidence le caractère démocratique de l'établissement des communes, et j'ai pensé que j'y réussirais mieux en quittant la dissertation pour le récit, en m'effaçant moi-même et en laissant parler les faits.'

Si la méthode change, le but aussi : il ne s'agit plus d'affirmer le pouvoir politique de l'industrie, mais de mener une réflexion sur la monarchie constitutionnelle. Les Lettres, en

${ }^{6} \mathrm{Ibid}$, p. $142-143$.

${ }^{7}$ A. Thierry, Lettres surl'histoire de France, Paris, Gamier, s.d. (Vers 1880), p. 4-5. 
contribuant «au triomphe des opinions constitutionnelles ${ }^{8}$, prennent place dans cette littérature libérale qui prépare la monarchie de Juillet. C'est dans cette perspective qu'A. Thierry donne une nouvelle analyse du mot «commune». Celui-ci n'est plus identifié à la classe industrielle, mais à la notion juridique de « constitution » :

De temporaires qu'elles étaient d'abord, ces associations de défense mutuelle, communions ou communes, comme on les appelait, devinrent permanentes; on s'avisa de les garantir par une organisation administrative et judiciaire, et la révolution fut accomplie. (...)

Ainsi le mot commune exprimait, il y a sept cents ans, un système de garantie analogue, pour l'époque, à ce que aujourd'hui nous comprenons sous le mot de constitution.'

La question qui préside aux développements sur l'affranchissement des communes confirme cette lecture politique: Louis le Gros a-t-il affranchi les communes? Cette interrogation vise finalement la Charte de 1814, où il est dit «que les communes ont dû leur affranchissement à Louis le Gros $»^{10}$. Il n'est d'ailleurs pas difficile de superposer la figure de Louis XVII à celle de son prédécesseur :

Louis le Gros devient ainsi, dans notre opinion, le promoteur de l'émancipation communale, le patron des libertés bourgeoises, le régénérateur du tiers état. Ces beaux titres lui sont même confirmés par le préambule de notre charte constitutionnelle; mais l'autorité de cette charte, souveraine en matière politique, est de nulle valeur en fait d'histoire. ${ }^{11}$

Il va donc falloir montrer que le mouvement des communes ne trouve pas son origine dans la volonté royale, mais dans un mouvement spontané, d'essence démocratique. Il ne s'agit ni d'octroi, ni de concession, mais de l'usage d'une force ouverte contre « la puissance établie $»^{12}$.

Pour étayer sa thèse, A. Thierry développe deux arguments différents. Il souligne tout d'abord le faible pouvoir du roi de France à l'époque, ainsi que les étroites limites de son royaume: l'eût-il voulu, il n'aurait pu affranchir qu'un nombre assez faible de communes. Le second argument consiste à affirmer que l'intérêt des bourgeois à se libérer des tutelles était bien plus important que l'intérêt qu'avait le roi à s' appuyer sur les villes pour combattre la puissance de ses vassaux :

Cette opinion se fonde $a$ priori sur l'intérêt qu'on suppose à Louis VI de faire de la puissance des bourgeois un contrepoids à celle des nobles; mais, en fait d'intérêt, la classe bourgeoise en avait un bien autre à l'érection des villes communes. On devrait donc, d'après cette manière d'argumenter, lui accorder encore la plus grande part dans la création de ce nouvel ordre de choses (...). Mais il ne s'agit pas d'argumentation logique, et l'histoire est là pour attester que, dans la grand mouvement d'où sortirent les communes ou les républiques du Moyen Age, pensée et exécution, tout fut l'ouvrage des marchands et des artisans qui formaient la population des villes. ${ }^{13}$

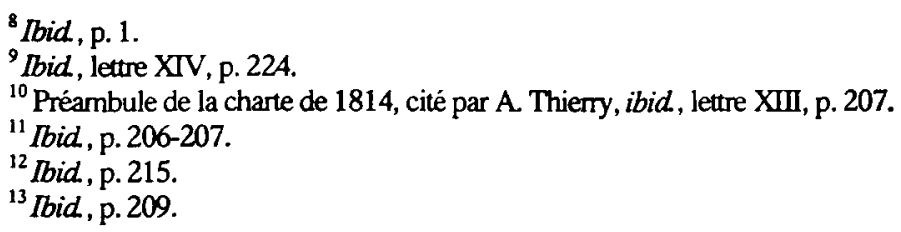


Une telle analyse est finalement conforme à l'esprit des théories saint-simoniennes: en refusant de prendre en réelle considération l'argument d'un conflit interne à la structure temporelle du Moyen Age - le roi contre les nobles - pour affirmer la pleine autonomie du mouvement des communes, A Thierry isole cette "révolution» du contexte temporel et spirituel dans lequel elle apparait. Il est clair que si le système féodal a une grande importance pour la formation de la France moderne ${ }^{14}$, il n'en a aucune, sinon de façon purement négative, dans l'affranchissement des communes.

C'est pour cette raison que la description des communes est constamment ramenée, par filiation ou par comparaison, à l'époque romaine. Pour caractériser les habitants des communes, la notion de classe laisse cependant la place à celle de race ou de mœurs :

Ce mouvement avait son foyer partout où subsistaient, depuis le temps des Romains, d'anciennes villes municipales. On eût dit que la race indigène, après avoir plié pendant cinq cents ans sous les institutions de la conquête, voulait, par un effort énergique, s'en affranchir $t$ les éloigner d'elle. Alors, il est vrai, la distinction primitive des races avait disparu, mais elle était en quelque sorte remplacée par la différence des moeurs; les pouvoirs du temps étaient marqués à l'empreinte des mours germaniques : le mépris pour la vie et la propriété des faibles, l'amour de la domination et de la guerre, formaient le caractère distinctif des seigneurs et des membres du haut clergé, tandis que le goût du travail et un sentiment confus de l'égalité sociale étaient, chez les habitants industrieux des villes, comme un débris de l'ancienne civilisation. ${ }^{15}$

Ces « héros de l'industrie naissante $\aleph^{16}$ sont en fait les héritiers, sinon de la race, du moins des mœurs romaines. L'importance accordée à la race pour penser l'histoire de France, est un des traits propres aux théories d'A. Thierry. Cette filiation absorbe même le caractère proprement social du mouvement, puisque « la classe bourgeoise ", ramenée à son origine, est identifiée à « la classe romaine ${ }^{17}$.

Les nouvelles institutions, pareillement, sont pensées en référence aux institutions romaines. En prenant pour principe les municipalités de la dernière période de l'empire romain, les communes retrouvent «l'enthousiasme républicain des vieux temps $»^{18}$. Les gouvemements électifs ressemblent aussi aux gouvernements municipaux des villes antiques. Enfin, le mouvement communal réussit surtout dans les villes du midi, où l'influence romaine était plus forte, et moins atténuée par la domination « franke".

Ayant pour objet d'affirmer la spontanéité de l'affranchissement des communes, la lecture d'A. Thierry fait dériver ce mouvement d'une source indépendante de ce qui constitue le cœur de la féodalité. Son analyse, plus attentive à l'amont qu'à l'aval, a en particulier du mal à situer ce mouvement par rapport à l'autre grand soulèvement démocratique: la révolution de 1789. Les comparaisons entre ces deux événements portent essentiellement sur la forme:

\footnotetext{
${ }^{14}$ Voir ibid., Letre IX, p. 135.

${ }^{15} \mathrm{Ibid}$, lettre XIV, p. 219.

${ }^{16} \mathrm{lbid}$., Lettre XXI, p. 337.

${ }^{17}$ Ibid, p. 219

${ }^{18}$ Ibid, letre XIII, p. 210.
} 
(...) j' ai spécialement en vue le caractère d'universalité et la marche pareillement progressive de ces révolutions, séparées d'ailleurs l'une de l'autre par de si énormes différences d'époque, de causes et de résultats politiques. ${ }^{19}$

Sur le fond, il y a deux points de rupture entre les deux révolutions. Le premier, qui s'accorde finalement avec les développements saint-simoniens, consiste à faire remarquer que la liberté à laquelle aspirent les communes n'est en rien comparable aux exigences de 1789. Il n'est alors question que «d'une liberté toute matérielle (...): la liberté d'aller et venir, de vendre et d'acheter, d'être maitre chez soi, de laisser son bien à ses enfants ${ }^{20}$. II s'agit, somme toute, de cette liberté minimale sans laquelle l'industrie n'est pas possible.

Le second point de rupture est évoqué dans la lettre XXV, « Sur l'histoire des assemblées nationales ». A. Thierry s'éloigne alors de Saint-Simon dans la mesure où l'affranchissement des communes n'est plus compris comme le premier pas d'une grande série qui mènerait jusqu'à la Révolution, et, au-delà, à la prise de pouvoir des industriels. Pour que se fasse sentir "le besoin d'une constitution générale ", il a fallu que l'autorité centrale énerve les constitutions municipales ${ }^{21}$. Or cette œuvre unificatrice et centralisatrice est due à l'un des éléments du pouvoir féodal: l'autorité royale. D'autre part entre l'affranchissement des communes et la Révolution, il faut introduire une étape intermédiaire: l'abolition de l'esclavage pour les campagnes, qui prend au quinzième siècle le relais des communes alors en déclin. Ainsi, la série industrielle, au sens où elle est définie par Saint-Simon, ne suffit pas même à rendre compte du mouvement général de l'histoire. La Lettre XXV indique clairement la nouvelle direction d'A. Thierry: l'histoire de France ne se résume plus aux progrès de l'industrie, mais à ceux du Tiers état.

\section{A. Comte.}

Le thème de l'affranchissement des communes est traité par Comte dès 1820 dans la Sommaire Appréciation de l'ensemble du passé moderne ${ }^{22}$. Cet opuscule, publié d'abord sous le nom de Saint-Simon dans l'Organisateur ${ }^{23}$, se ressent encore de l'influence du publiciste. Néanmoins, il est indéniable que la paternité de cet ouvrage revient à A. Comte, qui l'inséra d'ailleurs dans ses opuscules de jeunesse à la fin du Système de politique positive. Cette Sommaire Appréciation innove sur deux points essentiels par rapport aux travaux précédents.

- Tout d'abord cet exposé n'envisage pas le point de vue temporel indépendamment du point de vue spirituel. Au XI et au XII ${ }^{e}$ s., ce ne sont pas seulement les germes d'une nouvelle organisation temporelle qui apparaissent, mais aussi, grâce à l'introduction des sciences positives par les Arabes, ceux d'un nouveau système spirituel. De son côté, le Moyen Age

\footnotetext{
${ }^{19}$ Ibid, lettre XIV, p. 221.

${ }^{20} \mathrm{Ibid}$, p. 221.

${ }^{21}$ Ibid, lettre XXV, p. 413.

${ }^{22}$ A. Comte, Sommaire Appréciation de l'ensemble du passé moderne, Système de politique positive, Paris, 1929, appendice du t. IV, p. 4.

${ }^{23} \mathrm{H}$. Saint-Simon, $L$ 'organisateur (huitième et neuvième lettres), Guvres de Claude-Henri de SaintSimon, Genève, Slatkine reprints, 1977, t.II, p. 77.
} 
est définit comme la combinaison - c'est-à-dire comme le système - de deux pouvoirs. La féodalité ne peut donc plus être envisagée indépendamment du catholicisme, puisque chacun des éléments de la combinaison implique l'autre. De façon générale, la philosophie positive envisagera, sur le modèle du Moyen Age, tout système politique comme la combinaison du spirituel et du temporel.

- Cette Sommaire Appréciation, ensuite, esquisse un paradoxe qu'on ne trouve ni chez Saint-Simon, ni chez A. Thierry. Les germes de la nouvelle organisation politique apparaissent alors même que le système du Moyen Age atteint son développement intégral et définitif. Cette coïncidence, à vrai dire, n'est un paradoxe que pour les analyses précédentes, qui postulent l'hétérogénéité du mouvement des communes par rapport au Moyen Age. Au contraire, l'élaboration historique de Comte annule le paradoxe en montrant que la révolution communale est intimement liée au double système catholique et féodal.

Le Cours de philosophie positive reprend les résultats de la Sommaire appréciation en les complétant. Là où l'opuscule ne prenait en compte que l'évolution politique des sociétés modemes, le Cours envisage l'ensemble de l'histoire humaine. Dans la partie de cet ouvrage consacrée à la sociologie ${ }^{24}$, Comte est notamment conduit à étudier l'origine de l'affranchissement des communes et à apprécier la relation de ce mouvement avec le Moyen Age.

Les leçons du Cours qui abordent ces problèmes, rédigées entre 1840 et 1841 , sont plus tardives que les textes précédemment étudiés. Néanmoins, il s'agit de la première élaboration autonome de Comte relative aux communes. Par ailleurs, il faut préciser que le premier volume du Cours est paru en 1830 . On peut donc considérer que ces développements ont dans l'œurre de Comte le même statut que les Lettres de 1827 dans l'œuvre d'A. Thierry.

C'est dans la $56^{c}$ leçon que le problème de l'affranchissement des communes est directement évoqué. Il est cependant nécessaire de prendre en compte les deux leçons précédentes pour comprendre l'analyse comtienne.

Le Moyen Age, dont l'appréciation directe fait l'objet de la $54^{\mathrm{e}}$ leçon, présente une organisation politique bien supérieure à celle de l'antiquité, grâce notamment à la division du pouvoir en spirituel et temporel. Mais, bien qu'il soit « la vraie source générale de notre civilisation occidentale ${ }^{25}$, le système médiéval appartient encore à l'âge théologique et militaire. L'humanité ne peut passer directement de l'âge théologique et militaire, même envisagé dans sa forme ultime, à l'âge positif. C'est pourquoi la transition entre le Moyen Age et l'état positif est une opération complexe où deux mouvements sont à l'œuvre. Le premier mouvement est étudié dans la $55^{\circ}$ leçon. Purement transitoire, il consiste à désorganiser le système médiéval, sous le point de vue spirituel par l'action critique de la métaphysique, sous le point de vue temporel, par les légistes. Le second mouvement, qui fait l'objet de la $56^{\mathrm{e}}$ leçon, est organique: il est destiné à préparer «graduellement la société modeme à un système entièrement nouveau " ${ }^{26}$. Le premier élément de cette série organique

\footnotetext{
${ }^{24}$ La moitié du Cours est en effet consacrée à la sociologie. Comme il a été remarqué, cette sociologie est plus proche de ce que nous appelons aujourd'hui «philosophie de l'histoire " que du sens moderne du néologisme créé par Comte.

${ }_{25}^{25}$ A. Comte, Cours de philosophie pasitive, Paris, Hermann, 1975, t. II, 56 leçon, p. 493.

${ }^{26}$ Ibid., p. 483.
} 
concerne le nouveau pouvoir temporel: il s'agit de l'évolution industrielle ${ }^{27}$. C'est à l'origine de cette évolution que se place l'épisode des communes.

Pour analyser la période transitoire, caractérisée par ce double mouvement de désorganisation et d'organisation, A. Comte établit une chronologie un peu décalée par rapport aux repères historiques classiques. En effet, après sa constitution définitive et une période de stabilité, le Moyen Age se termine à la fin du XIII s. A partir du XIV s., commence la décomposition : celle-ci, sans entrer dans plus de détails, se compose elle-même d'une phase dite spontanée et involontaire, correspondant au $X T V^{\mathbb{E}}$ et au $X V^{\mathbb{E}}$ s., puis d'une phase systématique, appuyée par une doctrine négative (le protestantisme notamment), qui va jusqu'à la Révolution. L'évolution industrielle suit la même chronologie, à cette différence près, qu'il serait possible de faire remonter au XII ${ }^{e}$ s. l'origine du mouvement de recomposition. La raison de cette modification envisagée vient de ce que «la formation des classes nouvelles" précède leur première manifestation réelle ${ }^{28}$. Si le pouvoir politique de l'industrie se manifeste dès le début de la phase de décomposition spontanée, cette manifestation ne peut cependant se comprendre sans la formation préalable d'une nouvelle classe industrielle. Cela signifie que l'affranchissement des communes est à cheval sur deux périodes: les communes se constituent essentiellement pendant le plein essor du système médiéval (jusqu'à la fin du XII' ${ }^{\mathrm{e}} \mathrm{s}$.) pour affirmer leur pouvoir politique pendant la période de décomposition spontanée.

La chronologie du Cours confirme donc la coïncidence évoquée dans la Sommaire Appréciation. La formation de la classe industrielle, et son affranchissement se produisent à l'époque où le Moyen Age atteint son plein développement. Comte renverse donc complètement les thèses d'A. Thierry en montrant que le nouvel élément social est "essentiellement étranger à l'antiquité ${ }^{29}$, et dọit son essor à l'influence du système médiéval.

Ce renversement est surtout marqué dans la formation de la classe industrielle, dont la durée occupe finalement tout le Moyen Age. Comte distingue trois phases essentielles.

- La première phase consiste à passer de l'esclavage au servage. A cette transformation collaborent aussi bien la féodalité, en donnant une indispensable stabilité à la possession territoriale, que le catholicisme, en "imposant, avec une énergique autorité permanente, d'universelles obligations morales " ${ }^{30}$. C'est alors que la population agricole est incorporée à la société générale, et qu'apparaissent de "véritables droits sociaux, ne fût-ce que le plus élémentaire de tous, celui de former une famille ${ }^{31}$. Ce qui importe dans cette analyse, que l'on trouve plus ou moins chez Saint-Simon en 1817, c'est la signification que lui donne A. Comte. Le servage n'est pas la continuation de l'esclavage dans les campagnes, et n'a pas

27 Pour Comte, le pouvoir industriel est défini par l'action de l'homme sur la nature, alors que le pouvoir militaire est l'action oppressive de l'homme contre ses semblables. c'est pourquoi cette définition n'enveloppe pas seulement la population des villes, mais aussi la population agricole. De façon générale, la classe industriel est la classe laborieuse.

${ }^{28}$ Ibid., p. 483.

${ }^{29}$ Ibid., p. 493.

${ }^{30}$ Ibid.

${ }^{31}$ Ibid 
pour résultat de détourner l'attention des puissances féodales de la civilisation urbaine. Au contraire :

Une telle amélioration, base nécessaire de toutes les phases ultérieures d'émancipation civiles, me parait conduire, contre une opinion presque unanime aujourd hui, à placer dans les campagnes le siège initial de l'affranchissement populaire (...). ${ }^{32}$

- La deuxième phase, qui va du début du VI' $s$. jusqu'à celui du XI s., consiste dans l'abolition de la servitude populaire. L'accès à l'indépendance individuelle, servant de transition entre l'institution du servage et l'affranchissement des communes, «marque directement la différence la plus décisive entre la sociabilité moderne et celle de l'antiquité ${ }^{33}$. C'est le rachat volontaire de la liberté individuelle qui permet de donner « une organisation régulière " à l'initiation de la population à la vie industrielle, en lui faisant contracter des habitudes de modération et de prévoyance qui assureront son indépendance. Comme dans la première phase, les pouvoirs temporels et spirituels du Moyen Age ont contribué à ce changement. Mais cette étape se déroule d'abord dans les villes: l'affranchissement individuel sera plus tardif dans les campagnes. Sur ce point précis, Comte suit la thèse d'A. Smith concernant l'influence des villes sur les campagnes ${ }^{34}$. Dorénavant, les villes vont être le moteur de l'évolution industrielle.

Cette deuxième phase rompt donc définitivement avec toute interprétation qui tendrait à faire apparaitre l'affranchissement des communes comme la continuation des mours industrielles antiques.

-La troisième phase, qui commence au milieu du XI ${ }^{e}$ s., est proprement celle de l'affranchissement des communes. Au vu de ce qui précède, Comte reproche logiquement aux historiens modernes d'avoir méconnu l'influence du système catholique et féodal sur ce mouvement, et de l'avoir isolé de la phase précédente. L'ère des communes est en effet la simple continuation de l'émancipation personnelle. Comme dans les autres phases, les pouvoirs du Moyen Age contribuent au progrès de la classe laborieuse. La nature dispersive de l'organisme féodal permet d'inclure facilement « les communautés industrielles parmi les nombreux éléments de sa hiérarchie ${ }^{35}$. L'organisme catholique, quant à lui, voyait dans l'essor de classes nouvelles un moyen d'étendre sa domination et de maintenir l'indépendance du pouvoir spirituel. En affirmant la pleine adhésion du pouvoir sacerdotal à chaque étape du processus, A. Comte prend le contre-pied d'A. Thierry. Ce dernier, en effet, montre dans ses Lettres des conflits entre les communes et le clergé, dont la puissance, dans les villes du nord, est la dernière à résister à l'affranchissement.

L'analyse de la formation de la classe industrielle, composée des trois phases qui précèdent, rompt donc de manière patente avec les analyses antérieures. La période de décomposition spontanée du système médiéval, du début du $\mathrm{XIV}^{\mathrm{e}} \mathrm{s}$. jusqu'à la fin du $\mathrm{XV}$,

\footnotetext{
${ }^{32}$ Ibid.

${ }^{33}$ Ibid., p. 494.

${ }^{34}$ Voir A. Smith, Recherches sur la nature et les causes de la richesse des nations (les grands thèmes), éd. Gérard Mairet, Paris, Gallimard (coll. Folio), 1976, livre III, chap. 4, p. 214.
}

${ }^{35}$ A. Comte, Cours de philosophie positive, op. cit, p. 499. 
verra s'affirmer «l'irrévocable ascendant» de l'industrie ${ }^{36}$. La lutte interne au pouvoir féodal, opposant la noblesse au pouvoir royal, offre alors aux communes, grâce à un politique d'alliance, un espace politique suffisant.

Encore faut-il préciser à quelle liberté, et à quel pouvoir aspiraient les communes. La politique des classes laborieuses est alors « exclusivement industrielle ${ }^{37}$, et ne prétend pas aller au-delà "d'un essor suffisamment libre des facultés industrielles " ${ }^{38} .1 \mathrm{l}$ faut donc éviter toute assimilation entre cette liberté et celle des anciens, toute tournée vers la guerre:

(...) l'instinct vulgaire l'a ordinairement mieux appréciée jusqu'ici que la raison spéculative, qui, par un vicieux rapprochement, s'efforçait toujours de la subordonner à cette liberté politique particulière aux anciens, où l'esclavage des travailleurs constituait l'indispensable condition d'une turbulente participation de la caste guerrière à la direction journalière de ses affaires communes. ${ }^{39}$

De la même manière, A. Comte prend bien garde d'identifier «commune» et «constitution»: Ce serait confondre le pouvoir industriel avec celui des légistes, qui est critique, exclusivement appliqué à la destruction du pouvoir féodal, et non organique.

Dans la lecture d'A. Comte, l'affranchissement des communes n'est donc pas à proprement parler une période de rupture. Au contraire tout le Moyen Age a préparé l'avènement de la nouvelle classe industrielle. Aussi est-ce à tort que l'on a vu dans cet événement le point de départ radical de l'ère révolutionnaire:

Aussi ai-je cru devoir, pour la plus importante évolution élémentaire des sociétés modemes, spécialement rectifier d'abord une aberration fondamentale, qui, rompant tout à coup, dans le nœud le plus décisif, la continuité nécessaire de la progression humaine, empêche directement toute liaison vraiment philosophique du mouvement moderne au mouvement ancien. ${ }^{40}$

Ce que la Révolution de 1789 a mis en cause, c'est la cohérence de l'histoire. En reportant l'origine des mouvements révolutionnaires à la période des communes, on ne modifie pas la question, on la déplace simplement. En revanche, on fait porter sur le Moyen Age tout le poids du problème. Cet enjeu a certainement une part importante dans la fascination romantique pour cette période. C'est cette fascination qu'à leur manière, par l'évocations de rois aux noms gutturaux, ou à travers l'exaltation d'un système politique respectant l'indépendance du spirituel par rapport au temporel, A. Thierry et A. Comte illustrent. De bien riches illustrations, pour de si riches heures.

\footnotetext{
${ }^{36}$ Ibid., p. 510.

${ }^{37}$ Ibid., p. 506.

${ }^{38}$ Ibid., p. 505.

${ }^{39}$ Ibid., p. 506.

${ }^{40}$ Ibid., p. 500.
} 